\section{Buchrezension zu: Handbuch \\ Reisepharmazie 2021/22}

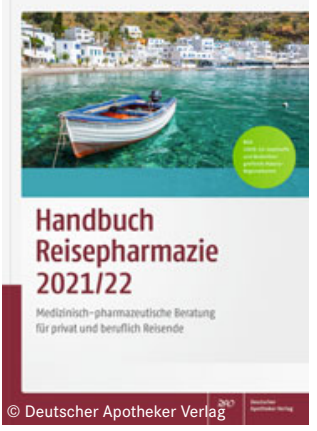

Handbuch Reisepharmazie 2021/22

Medizinisch-pharmazeutische Beratung für privat und beruflich Reisende

Christian Schönfeld

295 S., Deutscher Apotheker Verlag, 2021. SC, 49,80€.

ISBN: 9783769276084

DOI: $10.1007 / \mathrm{s} 12268-022-1699-4$

(C) Der Autor 2022

Die Corona-Pandemie hat allen sehr deutlich vor Augen geführt, dass Reisen in nahe und ferne Länder auch immer das Risiko der Übertragung von Erkrankungen beinhaltet. Zwar ist momentan die globale Reiseaktivität stark reduziert und der Fokus liegt ganz auf COVID-19, aber es gibt noch viel mehr Krankheitsrisiken, die in diesem Buch sehr übersichtlich und kompakt zusammengefasst werden.

Im ersten Teil werden systematisch die wichtigsten Reiseländer der Welt bezüglich empfohlener und notwendiger Impfungen und Krankheitsrisiken dargestellt. Ein Fokus liegt dabei auf der Malaria. Im zweiten Teil werden dann systematisch die wichtigsten Impfungen behandelt; auch die Impfstoffe gegen COVID-19 werden besprochen. Im dritten Teil behandelt das Buch die Malaria, im vierten Teil die wichtigsten Tropenerkrankungen und im fünften Teil werden allgemeine Hinweise zur Gesundheitsvorsorge bei Reisen gegeben. Für COVID-19 wird wegen der sich rasch ändernden
Infektionslage auf entsprechende aktuelle Webseiten verwiesen.

Das Buch ist ein kompaktes, aktuelles und systematisches Werk über die Reisemedizin mit einem besonderen Blick auf Infektionskrankheiten und Krankheitsprophylaxe. Die Therapie der Erkrankungen wird vermutlich aus Platzgründen nur kurz angerissen. Es kann als Nachschlagewerk jedem empfohlen werden, der eine Reise in nahe (und insbesondere ferne) Länder machen möchte. Das Buch ist eine sehr gute Erinnerung daran, dass es außer COVID-19 noch viele andere tückische Infektionskrankheiten gibt, die aber aktuell in der Öffentlichkeit praktisch nicht wahrgenommen werden. Preis und Ausstattung sind angemessen. Das Buch wird vor allem in tropenmedizinischen Beratungsstellen zum Einsatz kommen.

Roland Seifert, Medizinische Hochschule Hannover,

seifert.roland@mh-hannover.de

Diese Rezension erscheint Open Access. ${ }^{*}$

* Funding note: Open Access funding enabled and organized by Projekt DEAL. Open Access: Dieser Artikel wird unter der Creative Commons Namensnennung 4.0 International Lizenz veröffentlicht, welche die Nutzung, Vervielfältigung, Bearbeitung, Verbreitung und Wiedergabe in jeglichem Medium und Format erlaubt, sofern Sie den/die ursprünglichen Autor(en) und die Quelle ordnungsgemäß nennen, einen Link zur Creative Commons Lizenz beifügen und angeben, ob Änderungen vorgenommen wurden. Die in diesem Artikel enthaltenen Bilder und sonstiges Drittmaterial unterliegen ebenfalls der genannten Creative Commons Lizenz, sofern sich aus der Abbildungslegende nichts anderes ergibt. Sofern das betreffende Material nicht unter der genannten Creative Commons Lizenz steht und die betreffende Handlung nicht nach gesetzlichen Vorschriften erlaubt ist, ist für die oben aufgeführten Weiterverwendungen des Materials die Einwilligung des jeweiligen Rechteinhabers einzuholen. Weitere Details zur Lizenz entnehmen Sie bitte der Lizenzinformation auf

http://creativecommons.org/licenses/ by $/ 4.0 /$ deed.de. 